\title{
In Situ Nitroxide-Mediated Polymerized Poly(acrylic acid) as a Stabilizer/Compatibilizer Carbon Nanotube/Polymer Composites
}

\author{
Vitaliy Datsyuk, ${ }^{1}$ Laurent Billon, ${ }^{1}$ Christelle Guerret-Piécourt, ${ }^{1}$ Sylvie Dagréou, ${ }^{1}$ \\ Nicolas Passade-Boupatt, ${ }^{2}$ Sylvain Bourrigaud, ${ }^{2}$ Olivier Guerret, $^{2}$ and Laurence Couvreur ${ }^{2}$ \\ ${ }^{1}$ Equipe de Physico-Chimie des Polymères (EPCP), Institut Pluridisciplinaire de Recherche sur l'Environnement et \\ les Matériaux (IPREM), UMR 5254 CNRS, Université de Pau et Pays de l'Adour, Hélioparc Pau-Pyrénées, 2 Avenue Angot, \\ 64053 Pau Cedex 09, France \\ ${ }^{2}$ Groupement de Recherche de Lacq, ARKEMA, 64170 LACQ, France
}

Received 6 April 2007; Accepted 31 July 2007

Recommended by David Hui

\begin{abstract}
Carbon nanotube (CNT) polymer composites were synthesized via in situ nitroxide-mediated diblock copolymerization. Poly(acrylic acid) (PAA) was chosen as a first block to obtain a precomposite CNT-PAA which is readily dispersible in various solvents including water. The immobilization of the stable poly(acrylic acid) alkoxyamine functionality on the nanotube surface occurs during the synthesis of the first block without CNT prior treatment. The living character of this block is established by spectroscopic methods and the nature of the CNT/PAA interaction is discussed. This living first block offers the opportunity to reinitiate the polymerization of a second block that can be chosen among a wide range of monomers. This versatility is illustrated with a second block containing methyl acrylate (MA) or styrene (S). Scanning and transmission electron microscopies confirm good CNT dispersion in the polymer network, while transmission electron microscopy also spots the anchorage locations of PAA on the CNT surface. Such nanotubes wrapped by diblock copolymers can be dispersed in various polymer matrices to create CNT_-polymer composites. Conductivity measurements show that these composites obey a percolation-like power law with a low percolation threshold (less than $0.5 \mathrm{vol} \%$ ) and a high maximum conductivity (up to $1.5 \mathrm{~S} / \mathrm{cm}$ at room temperature).
\end{abstract}

Copyright (C) 2007 Vitaliy Datsyuk et al. This is an open access article distributed under the Creative Commons Attribution License, which permits unrestricted use, distribution, and reproduction in any medium, provided the original work is properly cited.

\section{INTRODUCTION}

The recently discovered carbon nanotubes (CNT) have attracted great interest as fillers for novel polymer composites [1-3]. CNT polymer composites are one answer among others to the increasing worldwide demand for novel polymer materials with improved physical and mechanical properties, for applications ranging from the aerospace domain to high-tech automotive components. For instance, in order to avoid electrostatic charging of an insulating polymer matrix or to apply electrostatic painting processes, sufficient electrical conductivity of the polymer is needed [4]. By using carbon nanotubes as fillers in those materials, one expects to introduce the required electrical conductivity together with a potential increase in mechanical performance. Tube diameters can range from 1 to $100 \mathrm{~nm}$ with aspect ratios (length/diameter) greater than 100 or 1000 .
The highly anisotropic nature of the tube is critical to provide percolative behavior at low-volume fractions, resulting in graphite-like electrical and thermal properties at 12 vol $\%$ additions [5]. Overall, CNTs are generally considered in three categories: single-wall nanotubes (SWNTs), double-wall nanotubes (DWNTs) and multiwall nanotubes (MWNTs). In order to take into account the wide range of available carbon nanotubes, we have started our work with different types of CNT, such as double-walled carbon nanotubes (DWNTs) and multiwalled carbon nanotubes (MWNTs).

CNT composites must be tailored in a way that will optimize the benefits of CNT in terms of mechanical, electrical, thermal, and rheological properties. This can be achieved only when the dispersion is well managed and for that, one must first be able to exfoliate efficiently the CNT which are initially arranged in much entangled faggots. Second, one 
must be able to avoid the agglomeration of the CNTs once dispersed. As for any fillers or pigments [6-8], the stability of the dispersion is obtained by wrapping the CNTs with polymers which will stabilize their dispersion-thanks to steric hindrance or electrostatic repulsion. However, it is always difficult to exfoliate nanoassembled systems with polymers since the gyration radius of the polymer is of the same order of magnitude as the voids in the faggots. Several approaches have been reported to prepare CNT polymer composites via exfoliation: solution processing [9], mesophase mediated processing, melt processing, and chemical modification of the CNT surface. Among the latter techniques, Lou et al. [10] on one side and Liu et al. [11] on another side reacted a living polystyrene chain obtained by nitroxide mediated polymerization (NMP). This "grafting to" technique is different from the in situ synthesis which is based on the direct growth of polymer chains in the CNT network [12-15]. The in situ route is taking advantage of the small dimensions of the starting monomers to swell the CNT network. Their polymerization provokes the exfoliation. This technique is similar to a "grafting from" method but in fact chemical grafting is not always necessary. Interestingly, no one yet tried to combine in situ CNT composite synthesis and NMP polymerization before our work.

Indeed, living polymerization is a very powerful technique to design polymer chains on the CNT surface. Reports of CNT composites synthesis using surface-initiated ring-opening polymerization [16] or surface-initiated atom transfer radical polymerization (ATRP) [17-19] have shown that those in situ living polymerization techniques are very efficient to disperse CNT in a polymer matrix. However, the functionalization of the CNT surface is always a complicated step which prevents any industrial development.

The aim of our work was thus to develop a simple method for the preparation of CNT-based polymer composites, via in situ nitroxide-mediated polymerization. This polymerization technique is industrially straightforward [20] and offers the possibility to polymerize most of functional vinylic monomers (styrenic, acrylic, and methacrylic) $[21,22]$.

Thus, using NMP to disperse nanotubes seems to be a new step towards the future development of nanotubecontaining materials.

The originality of our route comes not only from the fact that we were the first to combine NMP "in situ" polymerization and CNT but also because the block that we found to bring the most stable affinity with the CNT surface is a poly(acrylic acid) block $[23,24]$. Thus, it was often associated with a styrenic block supposedly bringing the affinity for the CNT surface $[18,25,26]$.

The paper here reports a thorough investigation of the different steps driving to those CNT diblock composites, trying to elucidate the nature and the reason of such strong interactions. In addition, in order to validate the fact that this method is not affecting the intrinsic expected properties of CNT composites, we studied the properties of such diblock CNT composites when blended in a polymer matrix.

\section{EXPERIMENTAL}

\subsection{Carbon nanotubes}

The double-walled carbon nanotubes (DWNTs) were synthesized by a catalytic chemical vapor deposition (CCVD) method [27] under hydrogen atmosphere with $18 \% \mathrm{~mol}$ of $\mathrm{CH}_{4}$ at $1000^{\circ} \mathrm{C}$, using Mo in addition to Co in an MgO-based catalyst, which was easily removed by a mild acidic treatment $(\mathrm{HCl})$. The DWNTs were washed with deionized water until neutrality, filtered, and dried overnight at $80^{\circ} \mathrm{C}$ in air. Statistical studies of TEM images of individual CNT have shown that most of them were double-walled and were 1-2 nm in diameter and more than $100 \mu \mathrm{m}$ in length.

The multiwalled carbon nanotubes (MWNTs) were provided by Arkema. They were also synthesized by a CCVD method and purified by acidic treatment. Statistical study of high-resolution electron microscopy images of the MWNT showed that $100 \%$ of them were multiwalled, with diameters ranging from 10 to $50 \mathrm{~nm}$.

1,4-dioxane, toluene, styrene, acrylic acid, and methyl acrylate were purchased from Aldrich and used without any further purification.

\subsection{Polymerization of the first block}

Carbon nanotubes (DWNTs and MWNTs) (0.2 g) were dispersed in $15 \mathrm{~mL}$ of 1,4-dioxane in a sonication bath for 1 hour, and $2 \mathrm{~g}$ of acrylic acid (AA) were then added. The reaction mixture was kept under stirring with a magnetic stirring barrel for 1 hour, and then sonicated for 1 additional hour. An alkoxyamine NMP initiator (2-methyl-2-[N-tert butyl$\mathrm{N}$-[diethoxy-phosphoryl-2, 2-di methyl propyl) aminoxy] propionic acid) (which is developed and commercialized by Arkema as Blocbuilder, and will be referred to as I in the remaining part of the paper) was then added with different ratios from $[\mathrm{AA}] /[\mathrm{I}]=50$ to $[\mathrm{AA}] /[\mathrm{I}]=300$. Nitrogen bubbling was used to deoxygenate the reaction mixture. Polymerization was performed at $115^{\circ} \mathrm{C}$. Finally, reaction mixture was cooled to room temperature. These conditions were directly derived from those elucidated by Couvreur et al. and used to form poly(acrylic acid)-based block copolymers by Laruelle et al. $[22,28]$. The net material obtained after that first polymerization step is termed as CNT-PAA precomposite in the remaining part of the paper.

\subsection{Copolymerization of the second block}

Respective amount of the second monomer (methyl acrylate (MA) or styrene (S)) was added to the reaction mixture of the CNT-PAA precomposite. The mixture was bubbled with nitrogen and heated to $115^{\circ} \mathrm{C}$. The net material obtained after this second step is named CNT-PAA-PM2 (nano-) composite. Two different composites are studied here: CNT-PAAPMA, with Poly (methyl acrylate) as a second block, and CNT-PAA-PS, with polystyrene as a second block. Note that the PM2 second block is in fact a statistical block resulting from the copolymerization of M2 monomer and the AA monomer remaining after the first block synthesis. However, 
the amount of remaining AA is less than $10 \mathrm{wt} \%$ compared to M2 monomer.

\subsection{Nuclear magnetic resonance}

NMR spectra were determined in solution of DMSO- $d_{6}$ in a Brucker advanced AM $400 \mathrm{MHz}$ spectrometer (frequency for ${ }^{31} \mathrm{P}$ phosphorus is $162 \mathrm{MHz}$ the chemical shifts are given in ppm relatively to a solution of $\mathrm{H} 3 \mathrm{PO} 4(85 \%)$ as the external reference).

\subsection{Scanning and transmission electron microscopies}

For SEM, an LEO1530 VP microscope has been used. Edges of the samples were observed after being cut with a diamond knife and were slightly recovered with a fine carbon layer to enhance local conductivity.

TEM observations were carried out on a Philips CM200 microscope. Ultra thin sections were directly cut from the films formed after drying of the as-synthesized CNT polymer composites, using an ultramicrotome and a diamond knife at room temperature. Sections were picked up on copper grids. To enhance contrast between PAA and PMA, the samples were stained by immersion in $\mathrm{RuO}_{4}$.

\subsection{X-ray photoelectron spectroscopy}

X-ray photoelectron spectroscopy (XPS) was performed using a Kratos 165 Axis Ultra.

\subsection{Thermogravimetrical analysis}

Thermogravimetrical studies were made on the TA Instruments TGA2950TA under nitrogen at $10^{\circ} \mathrm{C} / \mathrm{min}$ with temperatures ranging from 20 to $600^{\circ} \mathrm{C}$.

\subsection{Electrical conductivity measurements}

The conductivity measurements were performed at room temperature within the conventional four-wire probe configuration using a Keithey 6430 as current source and voltmeter. $\mathrm{V}(\mathrm{I})$ curves were generally ohmic on several decades.

\section{RESULTS AND DISCUSSION}

\subsection{Characterization of the CNT-PAA precomposite}

In our synthetic route (as described in $[23,24]$ and recalled in Figure 1), we used an in situ nitroxide-mediated polymerization with an alkoxyamine NMP initiator (I) that allows the synthesis of well-defined block or gradient copolymers with a good control of the polymer polydispersity [20].

Carbon nanotubes were dispersed in 1,4-dioxane which is a good solvent leading to stable CNT dispersions [23, 24] and also allowing for the control of the polymerization of the acrylic acid [28].

In order to understand the mechanism of the formation of the poly(acrylic acid) chains on the nanotube surface, monomer adsorption-desorption kinetics were studied. Results are presented in Figure 2.
TABLE 1: Correlation between AA adsorption and specific surface and oxygen content, determined by XPS, for different types of carbon nanotubes.

\begin{tabular}{lccc}
\hline CNT & BET $\left(\mathrm{m}^{2} * \mathrm{~cm}^{-3}\right)$ & {$[\mathrm{O}]$ at $\%$} & AA adsorption $\left(\mathrm{mg}^{*} \mathrm{~g}^{-1}\right)$ \\
\hline DWNT & 750 & 2.6 & 560 \\
MWNT & 150 & 0.6 & 70 \\
\hline
\end{tabular}

It appears from Figure 2 that the acrylic acid is rapidly adsorbed on the CNT surface. The amounts of adsorbed AA on both DWNT and MWNT, in 1,4-dioxane, are in strong correlation with the specific surface of nanotubes (determined by BET) and oxygen contents on their surfaces (determined by X-ray photoelectron spectroscopy) (see Table 1). It is known that this presence of oxygen is due to hydroxyl or carboxylic functions present at the surface of the CNT and usually generated by the acidic purification of the nanotubes [29]. Hence, one must assume that AA adsorbs on nanotubes via interactions between the acid function of the monomer and $-\mathrm{COOH}$ and/or $-\mathrm{OH}$ groups present at the CNT surface. This hypothesis is also supported by the results of Sonnenberg et al. [30] who found out that $\mathrm{COOH}-$ and/or $\mathrm{OH}-$ functional groups of substrates promote PAA adsorption.

In order to elucidate at what stage of the synthesis process the strong interaction between nanotubes and poly(acrylic acid) takes place, we compared the thermal degradation of a precomposite produced according to in situ synthesis (named CNT-PAA precomposite) and that of the samples of equivalent composition, obtained by mixing in solution a PAA prepared separately and CNT (named CNT-mixed PAA in what follows) (see Figure 3).

The thermogram of a pure PAA (see Figure 3 ) shows three decomposition stages which are well known [31]. The first decomposition stage (I), in the range of $50-180^{\circ} \mathrm{C}$, is attributed to the loss of absorbed water. The second decomposition stage (II), in an interval of $215-300^{\circ} \mathrm{C}$, corresponds to the dehydratation (anhydrification of vicinal acid functions) plus the decarboxylation of the polymer, which results in inter- and intramolecular bridging. The third decomposition stage (III), in the range of $365-470^{\circ} \mathrm{C}$, is a result of the final degradation of the polymer. Significant differences appear in decomposition tests of both CNT-containing samples at similar PAA amounts. CNT-mixed PAA showed behavior typical for the pure PAA with decomposition starting in the $50-180^{\circ} \mathrm{C}$ range. In this phase, the curves of pure PAA and CNT-mixed PAA are overlapping. In the case of the in situ polymerized CNT-PAA precomposite, we can observe that the polymer degrades at higher temperatures (onset at $250^{\circ} \mathrm{C}$ ), with a smooth threshold at $300^{\circ} \mathrm{C}$ and final decomposition occurring at $570^{\circ} \mathrm{C}$. This can be explained by strong interactions between CNT and polymer chains, which increases the thermal stability of the poly(acrylic acid). In their paper, Lou et al. reported a similar effect of MWCNT on polystyrene thermal degradation onset when polystyrene is chemically bonded to the tubes [10]. This also confirms what we already observed by XPS analysis $[23,24]$.

To understand deeper such a strong grafting, we compared the stability of a dispersion in water of a CNT-PAA 


\section{Precomposite CNT-PAA Composite CNT-PAA-PM2}

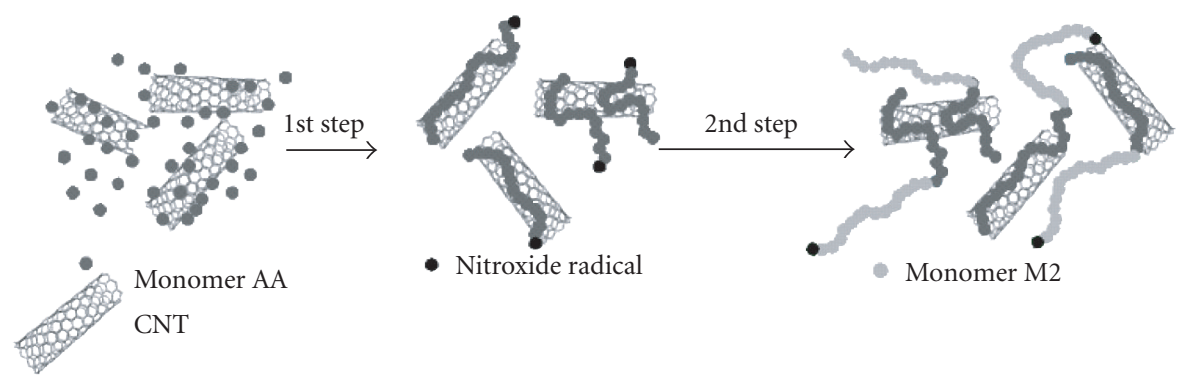

FIGURE 1: Schematic illustration of the composite preparation.

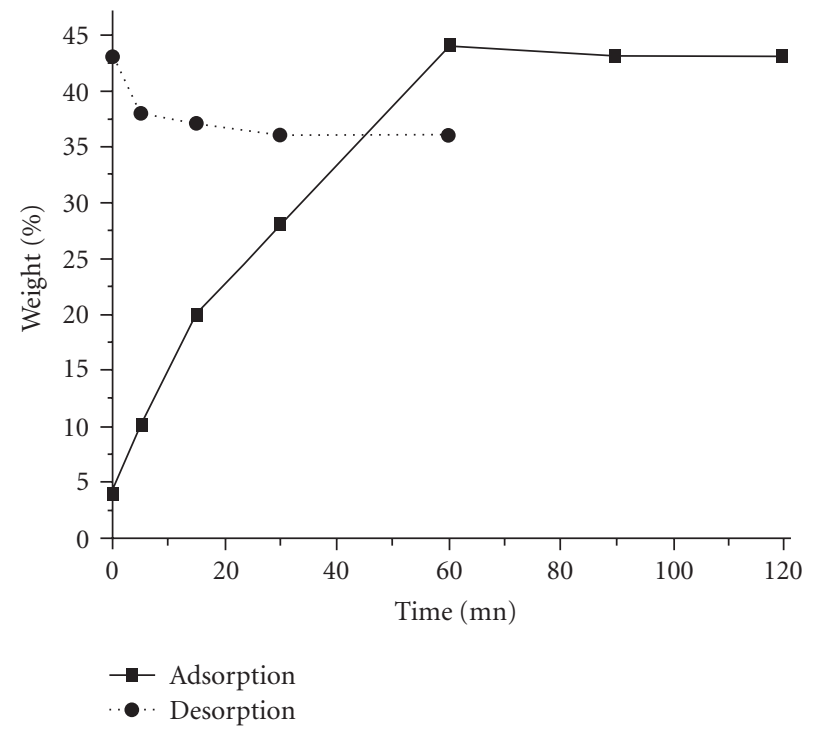

(a)

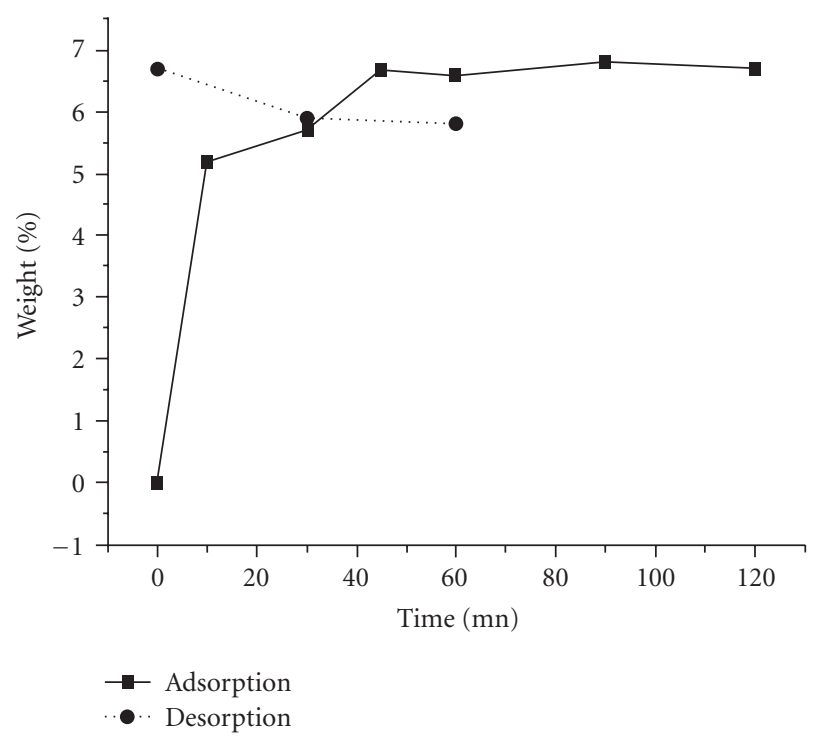

(b)

FIGURE 2: Adsorption-desorption kinetics of the acrylic acid on nanotube surface in dioxane (CNT concentration: $1 \mathrm{wt} \%$; AA concentration: 10 wt\%): (a) DWNT; (b) MWNT.

precomposite and a CNT-mixed PAA sample at $\mathrm{pH}=9$ (see Figure 4). The former leads to the formation of a long-time stable dispersion in water, while after 1 day, the latter sediments completely. This difference of behavior stresses once again the permanence of the CNT-PAA interactions which are generated during the in situ process. The reason why a simple mixing of PAA and nanotubes does not lead to similar interactions can be attributed to the fact that polymer chains are not able to penetrate the aggregates of nanotubes. Steric hindrance prevents any intereaction between polymer chains and chemical functionalities at the surface of the nanotubes, or creates an insufficient amount of interactions between macromolecular chains and the outer CNT. In the case of CNT-PAA precomposite, the chains immobilized at the surface of the CNT are generated in situ and form an electrostatic and steric barrier against agglomeration.

In order to fully characterize the CNT-PAA precomposites, we investigated the chain end of the poly(acrylic acid).
The interesting feature of the initiator (I) that was used in this work consists in the fact that it bears a phosphorus atom. Thus, it was possible to study chain ends by ${ }^{31} \mathrm{P}$ NMR (see Figure 5). The chemical shift of starting (I) is a single pick at $26.6 \mathrm{ppm}$ this is; a characteristic of an alkoxyamine where the nitroxide is attached to a tertiary nonchiral carbon atom ( $)$. When this alkoxyamine is a growing poly(acrylic acid) chain, the nitroxide is now linked to an enantiomeric secondary carbon atom $(\bullet)$, since the nitroxide moiety is also chiral $(*)$ (see Figure 5(b)). The resulting alkoxyamine is a mixture of two diastereoisomers with their own chemical shift. In Figure 5(b), we reproduced the ${ }^{31} \mathrm{P}$ NMR spectrum of the CNT-PAA precomposite, and we saw no longer a single pick in the region characteristic for alkoxyamines but two broad picks typical of the diastereoisomeric forms of the polyacrylic alkoxyamines. This NMR study on polymer chains of CNT-PAA precomposite is fully supported by previous work on nitroxide-mediated polymerization of acrylic acid reported by Lefay et al. [32]. Note that in this paper, the 


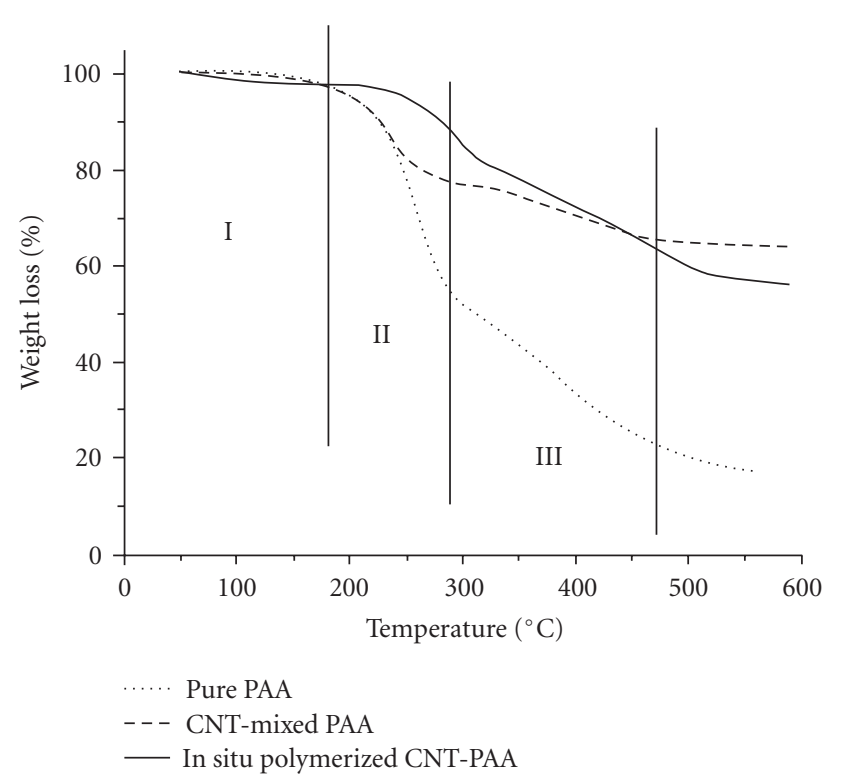

FIGURE 3: Thermogravimetric analysis (TGA) traces under nitrogen of pure PAA, MWNT-PAA precomposite, and MWNT-mixed PAA.

authors evaluated the amount of chains bearing a nitroxide to be above $85 \%$. Hence, we have spectrometric evidence that our precomposite is suitable to restart a second block polymerization.

\subsection{Effect of the initiator concentration}

Syntheses of CNT-PAA precomposites were performed by using two different initiator concentrations so that the monomer-to-initiator ratios were $[\mathrm{AA}] /[\mathrm{I}]=300$ and $[\mathrm{AA}] /[\mathrm{I}]=50$. Changing the initiator concentration is expected to vary the grafting density, the length of polymer chains, and the respective amounts of CNT and polymer chains in the CNT-PAA precomposites. Samples were tested by XPS (see Figure 6).

Figure 6 shows the deconvolution of the C1s peak into five main contributions. We focus our attention on the two most significant peaks: one at $284.2 \mathrm{eV}$ which is attributed to the graphitic carbon atoms of the nanotubes, and a second peak at $285.0 \mathrm{eV}$, which can be related to structural defects at the surface of the graphitic sheet. The low initiator concentration sample $([\mathrm{AA}] /[\mathrm{I}]=300)$ shows a strong peak at $284.2 \mathrm{eV}$, corresponding to the graphitization of the carbon nanotubes, and a low part of defect carbon atoms, as can be deduced from the low peak at $285.0 \mathrm{eV}$. On the other hand, one can see from the spectrum on the left that the sample with $[\mathrm{AA}] /[\mathrm{I}]=50$ shows the opposite trend, with a sharp decrease of the amount of graphitic carbon atoms on the sample surface, and an extreme increase of the carbon atoms in another state rather than the typical graphitic pure $\mathrm{sp}^{2}$ one. These results must be interpreted by keeping in mind that the XPS is a surface analysis method and may be explained by a difference of the polymer chains conformation onto the CNT surface. To confirm this hypothesis, a TEM study of the synthesized CNT-PAA precomposites was performed. TEM micrograms of both CNT-PAA precomposites are presented in Figure 7.

TEM images fully confirm our hypothesis about the difference in PAA organization onto the CNT surface. The precomposite synthesized with a high initiator concentration $([\mathrm{AA}] /[\mathrm{I}]=50)$ has a structure where poly(acrylic acid) encapsulates the carbon nanotubes. It is in good agreement with what was observed on the C1s XPS spectra, where the signal corresponding to nongraphitic carbon atoms is predominant. This signal can indeed be attributed to the carbon atoms of polymer chains that cover the CNT surface. On the other hand, PAA forms spots onto the CNT surface when polymerized with a low initiator concentration. In this case, the extreme surface of the precomposite is still dominated by the presence of graphitic carbon atoms from the CNT which explains the aspect of the XPS spectrum of this sample.

\subsection{Mechanism of grafting}

The NMR investigation revealed that most of the chains were terminated by an alkoxyamine. We initiated the polymerization of a second block PM2, which is built from a mixture of the remaining acrylic acid of the first block and another vinylic monomer (M2). Growing of the second polymer block PM2 thus led to gradual change of the hydrophilic feature of the final composite depending on the second monomer M2, promoting the good dispersion of the CNT in different polymer matrices. Study of the stability of the dispersions helped us in understanding the nature of the attachment of PAA to the CNT surface.

In this paper, we will discuss the cases where M2 is styrene (S) or methyl acrylate (MA). By varying the polymerization time of the second block, we obtained CNTPAA-PM2 composites with various second block lengths and different CNT amounts (from $0.9 \mathrm{wt} \%$ to $5.9 \mathrm{wt} \%$, according to thermogravimetric studies). We tried to determine the molecular weight of polymer chains by gel permeation chromatography (GPC) and we collected the supernatant of centrifuged CNT-PAA-PM2 composites diluted in acetone. Despite several washings in acetone, the resulting supernatant appeared slightly grayish, indicating the presence of grafted nanotubes and preventing the direct measurements of the molecular weight of the diblock copolymer by GPC [17]. For the moment, molecular weights of the different diblocks are supposed to be comparable to the one obtained in absence of CNT. The possibility to cleave selectively the linkage between CNT and diblock is still under investigation, in order to characterize precisely the grafted diblocks.

The stability of dispersions of various CNT polymer composites is summarized in Table 2. This study allowed us to determine the most probable mechanism of interaction between PAA chains generated in our process and the tubes.

The first possibility is the radical grafting of an alkoxyamine as reported both by Jérôme and Adronov (route I in Figure 8) $[10,11]$. If that was the only reason, then there 


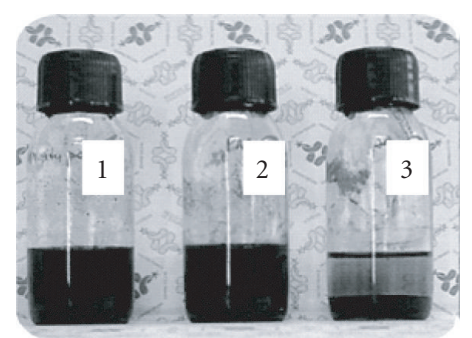

(a)

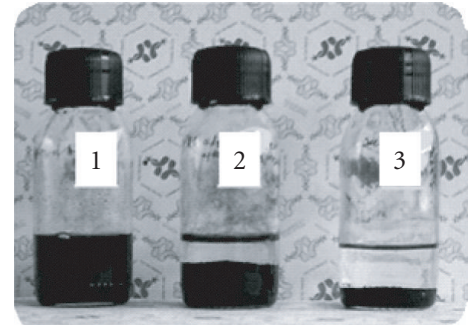

(b)

FIGURE 4: Stability of CNT water dispersions: (a) 1 hour after dispersion; (b) 1 day after dispersion. 1: in situ synthesized precomposite; 2 : CNT-mixed PAA; 3: pure CNT.

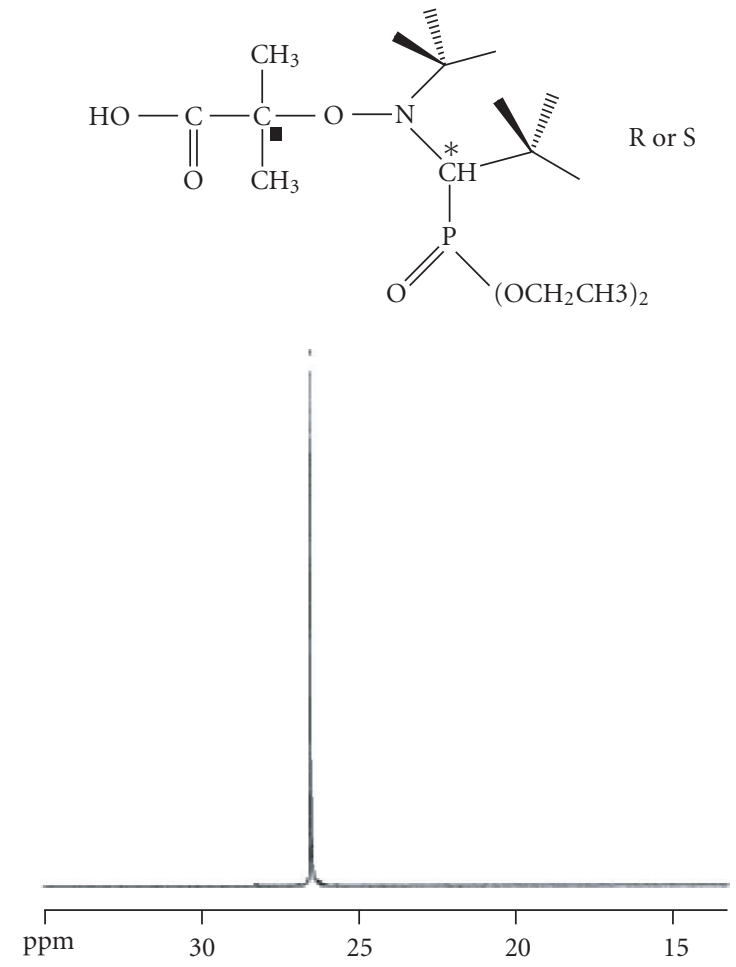

(a)<smiles>CCOP(=O)(OCC)[C@H](C(=O)O)N(O[C@H](C)C(=O)O)C(C)(C)C</smiles>

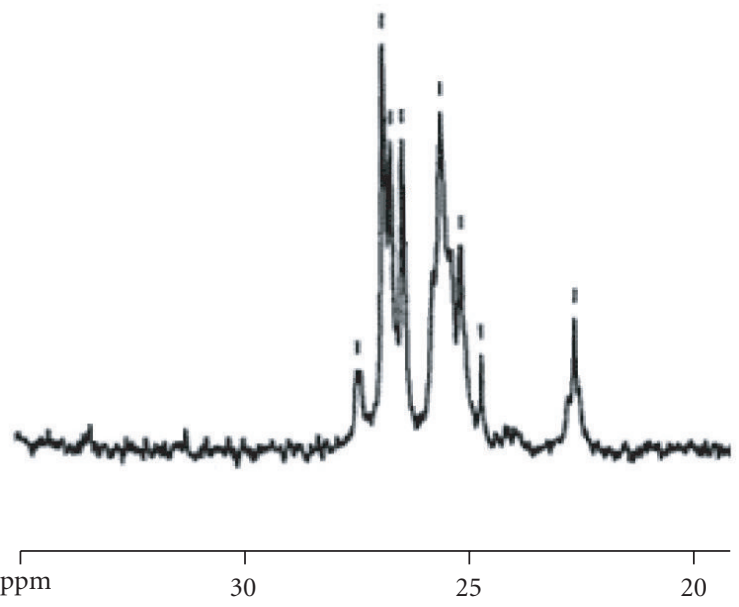

(b)

FIGURE 5: (a) ${ }^{31}$ P RMN spectra of initiator (I) and (b) of CNT-PAA precomposite, showing the presence of an alkoxyamine chain end.

should be only a minor difference between the grafting of a polystyrene (composite $\mathrm{C}$ ) and a polyacrylic alkoxyamine (composite B). We see in Table 2 that poly(acrylic acid) is bound to the CNT, whereas the polystyrene generated in the same conditions of temperature and dilution presents a nonpermanent interaction.

A second possibility (route II in Figure 8) corresponds to the grafting of acid functions of the polymer on the CNT surface. That is possible through esterification of the acid with alkoxy pendant functions on the CNT or through anhydrification with pendant acid functions. Such reactions are known to take place at elevated temperature, as reported by Baskaran et al. [33]. The NMP route that we chose presents the advantage of being processed at temperature above $110^{\circ} \mathrm{C}$ allowing such reactions.
The stability of dispersions of CNT-PAA-PS composite in toluene indicates two features. First, by comparing with the CNT/PS composite which does not provide a stable dispersion in toluene, we understand that the PAA first block induces strong attachment of the PS block on the CNT. Second, since the second block is well anchored, its growth has taken place from the surface of the tubes. This means that the starting PAA block grafted on the CNT had kept its livingness, indicating that the mechanism of grafting cannot be the radical coupling between the CNT and the living alkoxyamine. This also implies that a relevant amount of living PAA was grafted.

We cannot exclude the fact that route I occurs, but we estimate from these observations that route II is certainly the dominant mechanism of anchorage of the growing PAA on the CNT. 


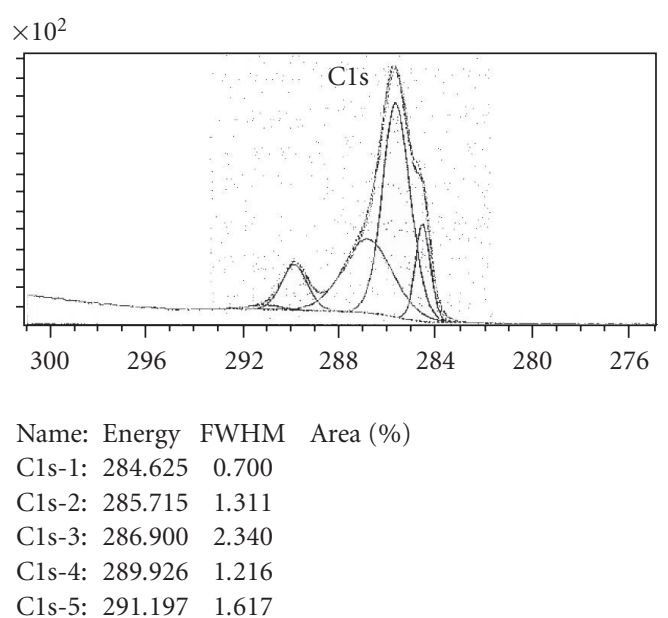

(a)

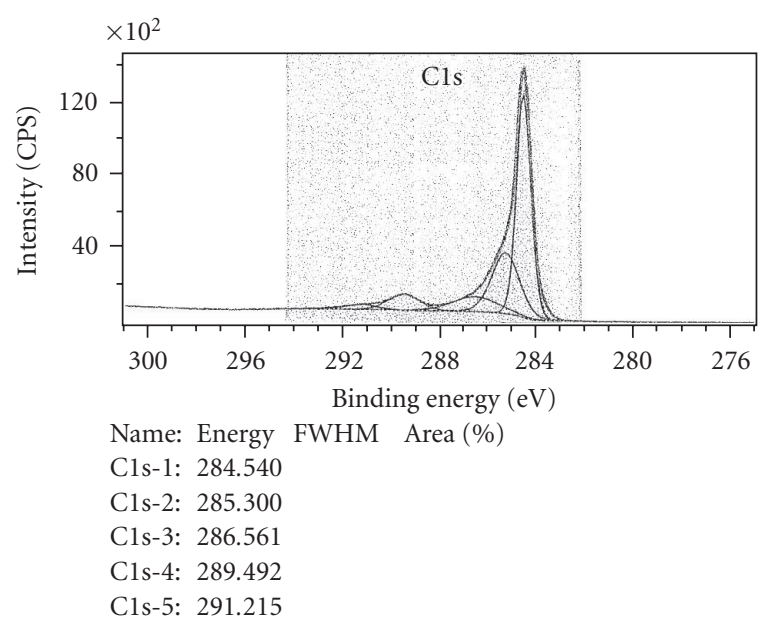

(b)

FIGURE 6: Deconvolution of the C1s peak of the CNT-PAA precomposites, synthesized with two different initiator concentrations: $[\mathrm{AA}] /[\mathrm{I}]=50$ and 300 (left to right, resp.).

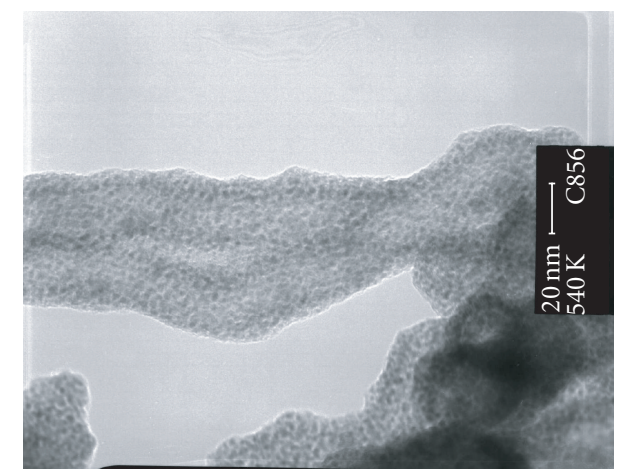

(a)

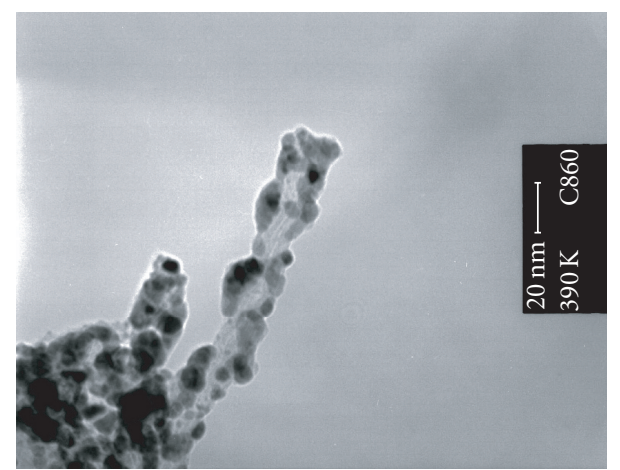

(b)

FIGURE 7: TEM micrographs of the CNT-PAA precomposites, synthesized with different initiator concentration: $[\mathrm{AA}] /[\mathrm{I}]=50$ on the left, and $[\mathrm{AA}] /[\mathrm{I}]=300$ on the right $($ scale bare $=20 \mathrm{~nm})$.

\subsection{Electron microscopy of the CNT-PAA-PM2 composite}

While the first block of poly(acrylic acid) afforded a good dispersion ability of the CNT in water, by being able to restart a second polymerization from the alkoxyamine chain end, we were able to vary the affinity of the nanotubes with all kinds of hydrophobic media. At this point, we chose to focus our study on the dried material directly in order to characterize the composites. We led microscopy investigations and then electrical measurements on cast films obtained by slow evaporation of a dioxane solution of the composite.

The CNT-PAA-PM2 composite films were observed at different scales. Figure 9 shows SEM micrographs of MWNT-PAA-PMA and MWNT-PAA-PS composites, respectively, containing $3.9 \mathrm{wt} \%$ and $5.9 \mathrm{wt} \%$ of carbon nanotubes. On these micrographs, MWNTs appear as shining points. On both samples, one can observe some areas of relatively high concentrations of carbon nanotubes in the composites. However, apart from these clusters, the nanotubes are dispersed uniformly in the polymer matrices but they are difficult to image due to their small size. Such structures of CNT dispersed in polymer have been observed in the literature [34]. Field emission gun scanning electron microscopy (EFG-SEM) is in progress to characterize the samples containing DWNT, because of the better resolution necessary to image DWNT.

To investigate the dispersion at a nanometer scale and to characterize the anchorage of the polymer on the nanotube surface, TEM was used. First, in Figure 10, we confirm that the carbon nanotubes, initially forming aggregates, are isolated after the copolymer synthesis. Figures 10(a) and 10(b) reveal the nanostructuring of the PAA-PMA diblock (respective contents in the diblock are about $10 \mathrm{wt} \%$ and $90 \mathrm{wt} \%$ ) in the presence of carbon nanotubes. Thanks to $\mathrm{Ru}_{4}, \mathrm{PAA}$ chains are indeed stained preferably and appear to be concentrated in nanospheres (black areas) whose sizes are in the order of magnitude of ten nanometers. Note that the micrographs (see Figure 10(c)) of pure PAA-PMA synthesized in the same way but in the absence of CNT they show a 
TABLE 2: Stability of CNT-based composites in appropriate solvents.

\begin{tabular}{lcccc}
\hline Composite & CNT+ PAA (A) & CNT-PAA (B) & CNT-PS (C) & CNT-PAA-PS (D) \\
\hline Process & Blend & In situ & In situ & In situ \\
Stability* & No & Yes (water) & No (toluene) & Yes \\
\hline
\end{tabular}

* of the dispersion in appropriate solvent.

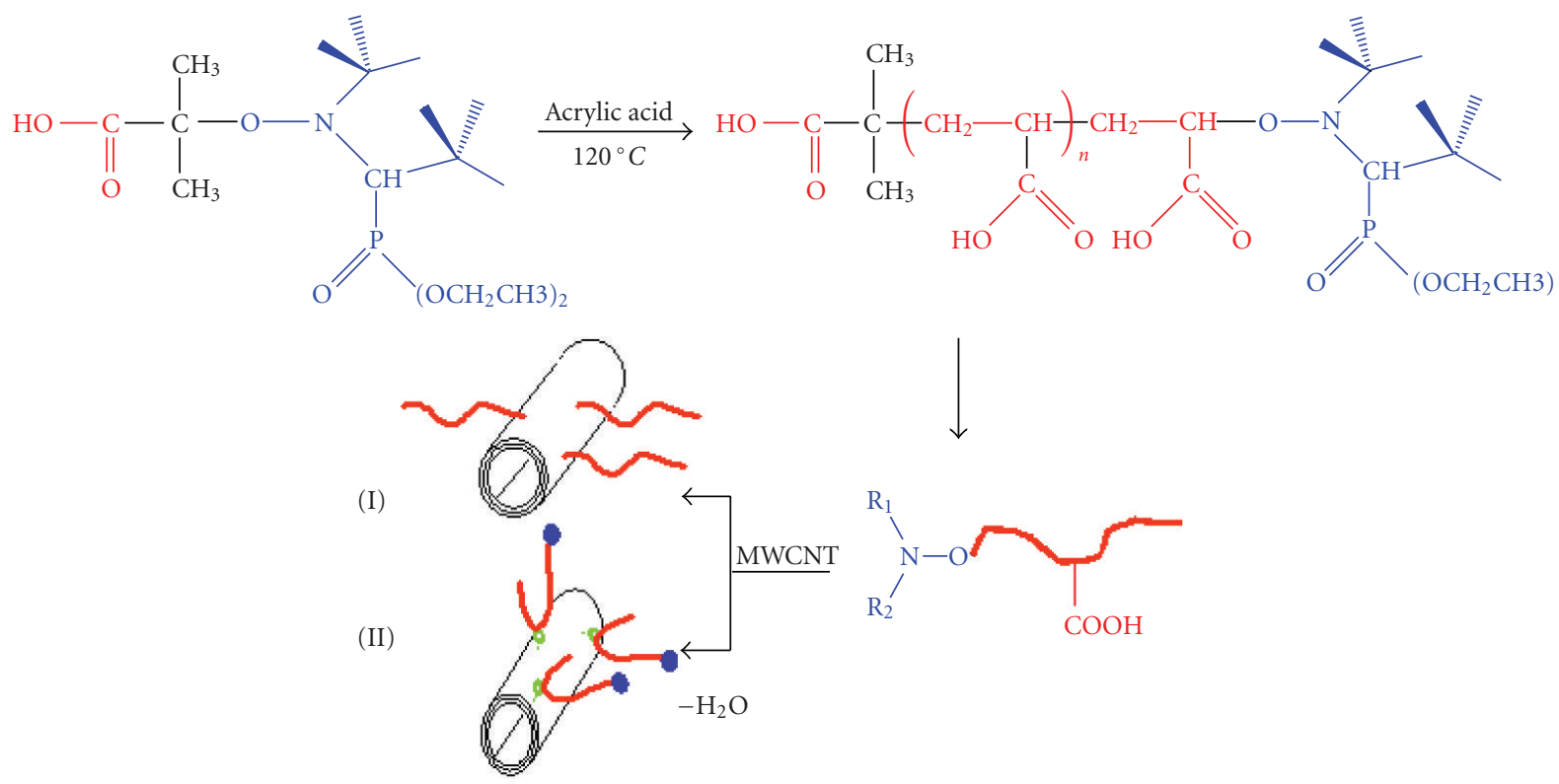

FIGURE 8: Possible mechanisms of grafting of NMP growing polyacrylic acid (I) by radical reaction of the macroalkoxyamine on the CNT conjugated surface or (II) by esterification or anhydrification of acid functions (red) with pendant alcohol or acid functionalities (green circles).

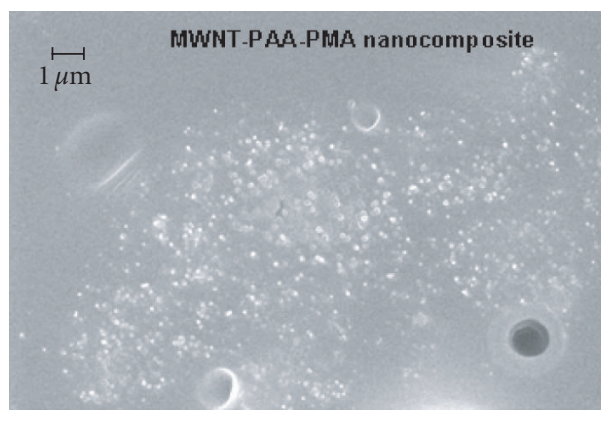

(a)

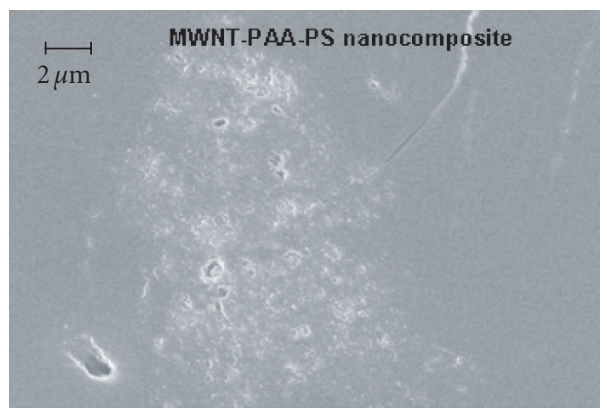

(c)

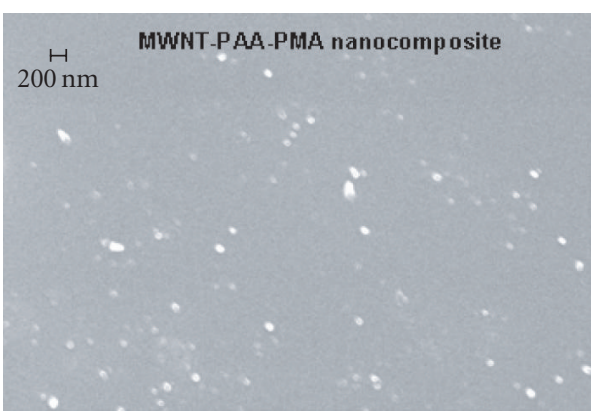

(b)

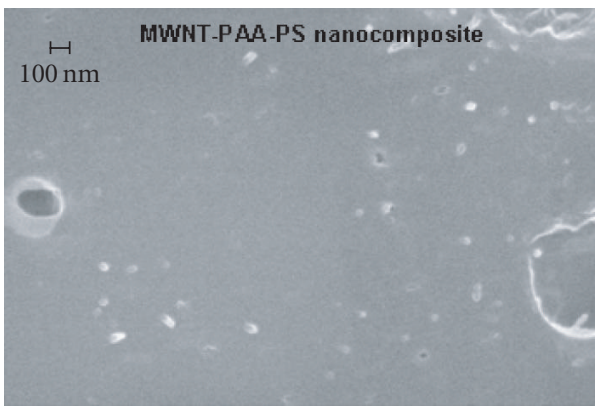

(d)

FIgURE 9: Scanning electron microscopy images of the MWNT-PAA-PMA and MWNT-PAA-PS composites. 
very similar nanostructuring of the diblock copolymer. Such nodular organization is expected for the range of composition that we have explored (from $4 \%$ to $10 \%$ of PAA in the diblock). The presence of CNT during the polymerization does not change the diblock organization in this window of block ratio.

Figure 10(b) shows a very exciting feature since at this level of magnification, we can easily point out domains of PAA which are regularly spotted on the surface of a nanotube. Those domains indicate the sites of interaction between the nanotube and the polymer chains. Contrary to what is generally assumed in the literature, we see here that the grafting is discrete and that it does not fit with a model of continuous wrapping of the surface. We are currently working on measuring the spacing of those domains which should be correlated to the average distance between oxygen atoms present at the surface of the nanotube.

\subsection{Conductivity of the CNT-PAA-PM2 composites}

The electrical conductivities of the composites CNT-PAAPM2 were measured for different lengths of the PM2 second block. In all cases, whatever the second block, PMA, or PS is, and whatever the CNT, DWNT or MWNTs, we observed a sharp increase of the electrical conductivity when increasing the CNT amount and a saturation behavior at large CNT amounts. This is the signature of a percolation behavior.

As an example, from the same precomposite DWNTPAA (whose CNT content was $32 \mathrm{wt} \%$ ), a second block of PMA was polymerized for three different polymerization times (respectively, 30, 120, and 160 minutes) leading to three different final amounts of CNT (respectively, $4.7 \mathrm{wt} \%, 1.9 \mathrm{wt} \%$, and $1.5 \mathrm{wt} \%)$ in the DWNT-PAA-PMA composites. Their respective conductivities were $0.59 \mathrm{~S} / \mathrm{cm}$, $0.22 \mathrm{~S} / \mathrm{cm}$, and $10^{-5} \mathrm{~S} / \mathrm{cm}$, showing that the composites reach very high conductivities compared to the classical conductivity value of polymers (below $10^{-14} \mathrm{~S} / \mathrm{cm}$ for PMA and PS). Note that the conductivity of pressed pellets of pure DWNT was found around $25 \mathrm{~S} / \mathrm{cm}$ at room temperature, and the one of DWNT-PAA precomposite was around one order of magnitude less. These results show that above $1.9 \mathrm{wt} \%$, the composites conductivity was dominated by the DWNT-PAA or by the DWNT mat conductivities.

The measured conductivity should follow a percolationlike power law near the threshold $\sigma=\sigma\left(x-x_{c}\right)^{t}$, where $x$ is the volume fraction of the conductive filler, $x_{c}$ is the percolation threshold, and $t$ is the conductivity critical exponent. In the composites, the mass fraction is related to the volume fraction through the ratio of the density of the polymer matrix over the density of CNT. The density of MWNT is usually estimated to $2 \mathrm{~g} / \mathrm{cm}^{3}[34,35]$, and the densities of PS and PMA are close to $1 \mathrm{~g} / \mathrm{cm}^{3}$, so that what follows, we consider that the volume fraction is around twice less than the mass fraction. Note that this assumption is probably not a correct estimation in the case of DWNTs that are aggregated in bundles for which density is smaller (around $1.3 \mathrm{~g} / \mathrm{cm}^{3}$ for SWNT bundles).

In the case of CNT-PAA-PM2 composites, with different lengths of the second block PM2, it is difficult to consider a percolation law because the polymer matrices are too different depending on the polymerization time used for the second block. Indeed, for the DWNT-PAA-PMA (30 minutes) sample, the ratio of PAA/PMA is around 10 units/90 units determined by NMR ${ }^{1} \mathrm{H}$, whereas for DWNT-PAA-PMA (160 minutes) the ratio is 10 units/280 units, so that we can hardly consider that the matrix is still the same.

\subsection{Dilution of CNT-PAA-PM2 composites in a polymer medium}

The last step that we can achieve to prepare CNT composites consists in diluting CNT-PAA-PM2 composite in a polymer for which PM2 is a compatible block. We chose to blend CNT-PAA-PMA with an emulsion of poly(methyl acrylate) (EPMA), while CNT-PAA-PS was solvent-mixed (1,4-dioxane) with high molecular weight polystyrene PS. EPMA was synthesized in laboratory by typical emulsion polymerization methods, using a sodium persulfate as an initiator and nonionic surfactant TRITON X 400. This example was chosen to illustrate the possibility of introducing CNT into aqueous acrylic paints. The composites obtained through these blends were then dried by slow evaporation to cast a film. The films that we obtained will be referenced as CNT-PAA-PMA/EPMA and CNT-PAA-PS/PS. They both showed a good CNT distribution (checked by TEM and SEM images were not shown).

In our experimental setup, the conductivity range is limited to values above $10^{-8} \mathrm{~S} / \mathrm{cm}$. Once again, the conductivity curves, shown in Figure 11, reveal percolation behaviors. The percolation threshold is in both cases estimated at around $0.5 \mathrm{vol} \%$, and the maximum conductivity is above $1 \mathrm{~S} / \mathrm{cm}$. Because of the few number of points, no determination of the parameters $\sigma_{0}, x_{c}$, and $t$, using a least square method, was possible. However, the gray tendency curves shown in Figure 11 are used as the best fit of the experimental values. They correspond, respectively, to the following equations: $\sigma=1.14(x-0.5)^{2}$ and $\sigma=0.1(x-0.27)^{2}$. In both case, the critical index $t=2$ is close to $t=1.94$, that is, the value predicted by the percolation theory for $3 \mathrm{D}$ composites [36]. Note also the low values of the threshold, 0.5 and 0.27 , that confirm the interest in these composites as conductive fillers in different polymer matrices. In the case of MWNT-PAAPS blended with PS, even for low content (10\% of MWNTPAA-PS for $90 \%$ of industrial PS), the conductivity of the resulting composite is very important (around $10^{-3} \mathrm{~S} / \mathrm{cm}$ ) [24].

\subsection{Application of the MWNT-PAA precomposites to "in situ" emulsion polymerization}

We finally discuss the example of the use of the poly(acrylic acid)-modified carbon nanotubes in in situ emulsion polymerization. Series of the MWNT poly(styrene-co-butyl acrylate) composites were synthesized by emulsion polymerization, in the presence of MWNT-PAA precomposites. We varied the amount of MWNT in the final MWNT poly(styreneco-butyl acrylate) composites from 0.7 to $4 \mathrm{wt} \%$. The concentration of the polymers in emulsion ranged from 24 to 


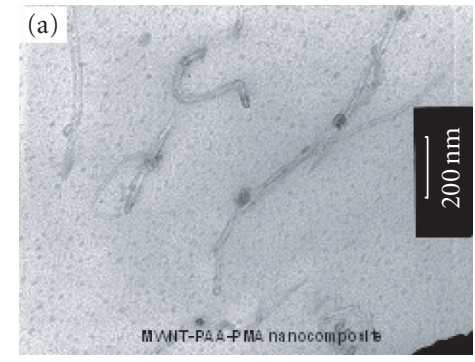

(a)

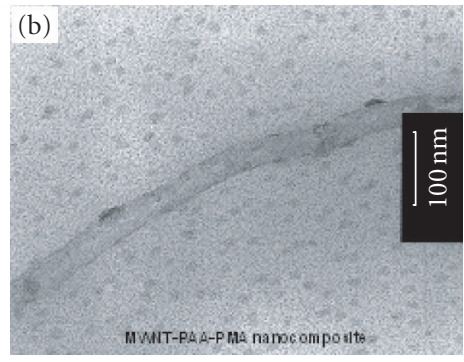

(b)

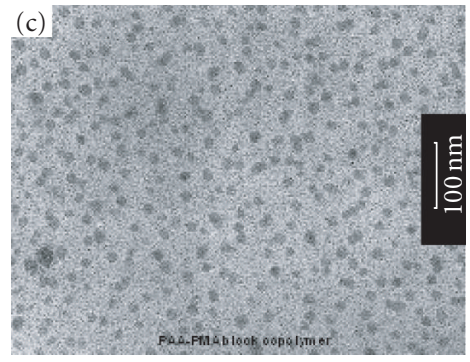

(c)

FIGURE 10: Transmission electron microscopy images of the MWNT-PAA-PMA composite. Black areas correspond to regions stained by $\mathrm{Ru}_{4}$, where PAA is dominant.

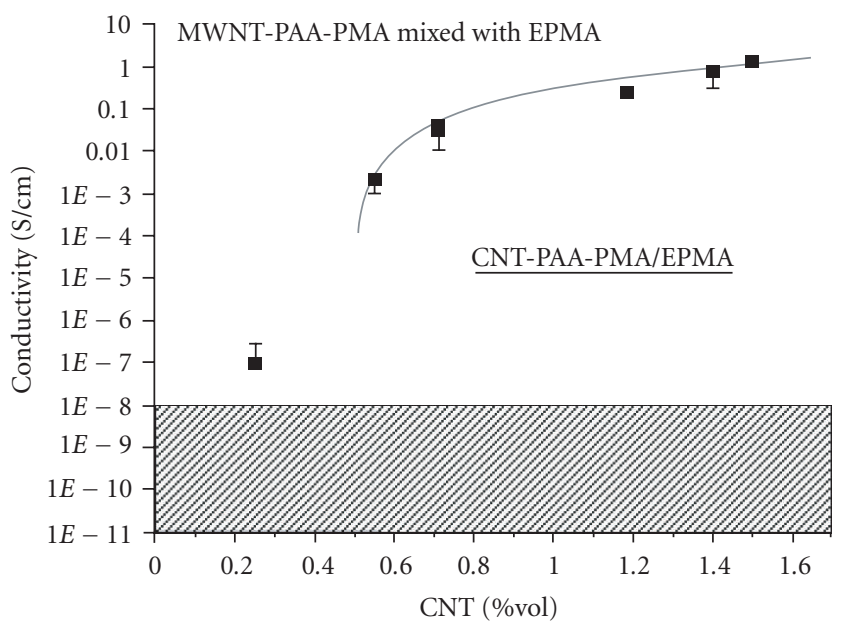

(a)

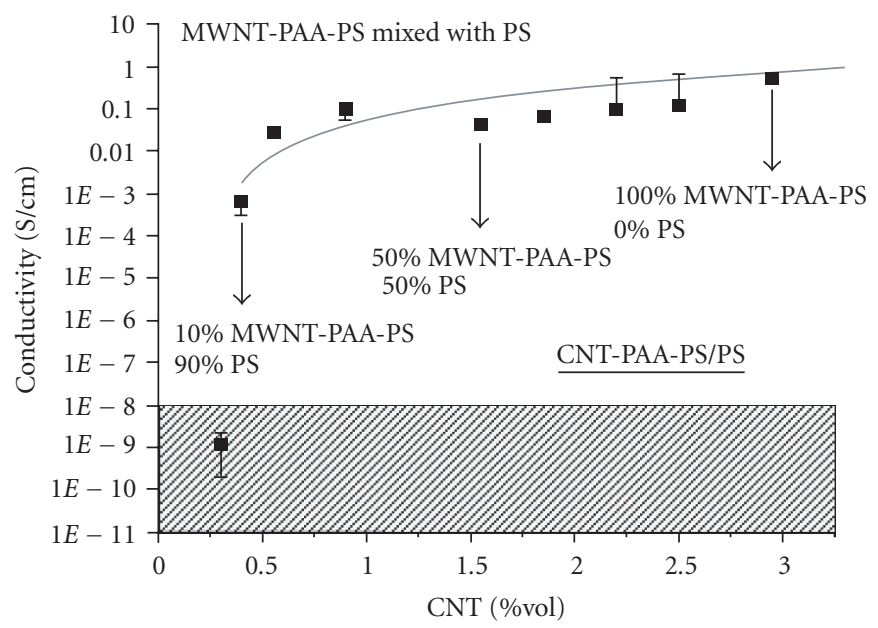

(b)

FIGURE 11: Four-wire conductivity measurements of the composites CNT-PAA-PMA/EPMA and CNT-PAA-PS/PS. Dashed areas correspond to conductivity regions that are not accessible with our apparatus.

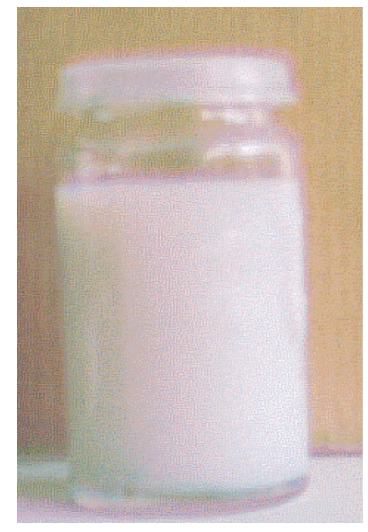

(a)

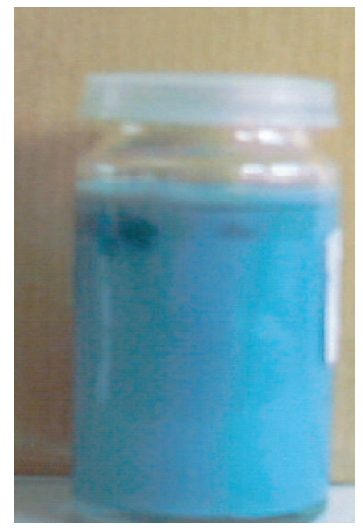

(b)

FIgURe 12: Poly(styrene-co-butyl acrylate) emulsion synthesized without (1) and in presence (2) of MWNT-PAA precomposite.

$45 \%$ wt. Monomers were used under the ratio $[\mathrm{S}]:[\mathrm{BA}]=$ 1:1. The nonionic surfactant Igepal CO 890 was used as an emulsifier, and the potassium persulfate was the initiator. Polymerization was performed at $70^{\circ} \mathrm{C}$. Figure 12 reports the photos of the obtained latexes.
The studies of MWNT polymer composites synthesized by in situ emulsion polymerization are underway but the synthesized carbon nanotubes-filled polymer composites show a good stability in emulsion and can be used as a basis for the preparation of conductive paintings and coatings. 


\section{CONCLUSION}

We report the synthesis of the CNT-PAA-PMA and CNTPAA-PS composites via in situ nitroxide-mediated polymerization. NMP has been successfully applied for polymerization of the block copolymer, where the first block of poly(acrylic acid) is strongly bound to the CNT, and the second block has been efficiently used as a compatible block to disperse CNT in a selected matrix. Anchorage of the block copolymer at the surface of the tubes is a specificity of the in situ polymerization of acrylic acid at elevated temperature. TEM microscopy reveals that, contrary to what is generally assumed in the literature, the grafting is discrete. It does not fit a model of continuous wrapping of the surface; the grafting should thus be due to the presence of much localized spots on CNT. This fact might be related to the percolation effect that we observed by electrical conductivity measurement, since the polymer does not isolate the nanotubes and therefore does not prevent the electronic movement through the tubes. Moreover, this approach can be extrapolated to emulsion process in order to elaborate a facile route to CNTdispersed latex for conductive films or coatings applications.

\section{ACKNOWLEDGMENTS}

We are grateful to E. Flahaut, C. Laurent, and A. Peigney from CIRIMAT (Toulouse, France) for supplying the DWNT. We thank Arkema for supplying the initiator I (Blocbuilder), MWNT, high molecular weight PS Lacqrene 1160, and performing the TEM measurements, with B. Pouchan-Lahore. We also thank Arkema for financial support (VD). We acknowledge J.C. Dupin from LCTPCM (Pau, France) for performing the XPS measurements and A. Khoukh for carrying out the RMN measurements. This work has been done in the framework of the GDRE n ${ }^{\circ}$ 2756. "science and applications of the nanotubes NANO-E."

\section{REFERENCES}

[1] H. D. Wagner, O. Lourie, Y. Feldman, and R. Tenne, "Stressinduced fragmentation of multiwall carbon nanotubes in a polymer matrix," Applied Physics Letters, vol. 72, no. 2, pp. 188-190, 1998.

[2] R. Andrews, D. Jacques, A. M. Rao, et al., "Nanotube composite carbon fibers," Applied Physics Letters, vol. 75, no. 9, pp. 1329-1331, 1999.

[3] D. Qian, E. C. Dickey, R. Andrews, and T. Rantell, "Load transfer and deformation mechanisms in carbon nanotubepolystyrene composites," Applied Physics Letters, vol. 76, no. 20, pp. 2868-2870, 2000.

[4] J. Sandler, M. S. P. Shaffer, T. Prasse, W. Bauhofer, K. Schulte, and A. H. Windle, "Development of a dispersion process for carbon nanotubes in an epoxy matrix and the resulting electrical properties," Polymer, vol. 40, no. 21, pp. 5967-5971, 1999.

[5] E. W. Wong, P. E. Sheehan, and C. M. Lieber, "Nanobeam mechanics: elasticity, strength and toughness of nanorods and nanotubes," Science, vol. 277, no. 5334, pp. 1971-1975, 1997.

[6] L. Ghannam, H. Garay, M. E. R. Shanahan, J. François, and L. Billon, "A new pigment type: colored diblock copolymer-mica composites," Chemistry of Materials, vol. 17, no. 15, pp. 38373843, 2005.
[7] R. Inoubli, S. Dagréou, A. Khoukh, F. Roby, J. Peyrelasse, and L. Billon, “'Graft from' polymerization on colloidal silica particles: elaboration of alkoxyamine grafted surface by in situ trapping of carbon radicals," Polymer, vol. 46, no. 8, pp. 24862496, 2005.

[8] H. Yokoyama, T. Miyamae, S. Han, et al., "Spontaneously formed hydrophilic surfaces by segregation of block copolymers with watersoluble blocks," Macromolecules, vol. 38, no. 12, pp. 5180-5189, 2005.

[9] R. Shvartzman-Cohen, E. Nativ-Roth, E. Baskaran, Y. LeviKalisman, I. Szleifer, and R. Yerushalmi-Rozen, "Selective dispersion of single-walled carbon nanotubes in the preence of polymers: the role of molecular and colloidal length scales," Journal of American Chemical Society, vol. 126, no. 45, pp. 14850-14857, 2004.

[10] X. Lou, C. Detrembleur, V. Sciannamea, C. Pagnoulle, and R. Jérôme, "Grafting of alkoxyamine end-capped (co)polymers onto multi-walled carbon nanotubes," Polymer, vol. 45, no. 18, pp. 6097-6102, 2004.

[11] Y. Liu, Z. Yao, and A. Adronov, "Functionalization of singlewalled carbon nanotubes with well-defined polymers by radical coupling," Macromolecules, vol. 38, no. 4, pp. 1172-1179, 2005.

[12] R. Haggenmueller, H. H. Gommans, A. G. Rinzler, J. E. Fosher, and K. I. Winey, "Aligned single-walled carbon nanotubes in composites by melt processing methods," Chemical Physics Letters, vol. 330, no. 3-4, pp. 219-225, 2000.

[13] E. Kymakis, I. Alexandou, and G. A. J. Amaratunga, "Singlewalled carbon nanotubes-polymer composites: electrical, optical and structural investigation," Synthetic Metals, vol. 127, no. 1-3, pp. 59-62, 2002.

[14] Y. Lin, B. Zhou, K. A. Shiral Fernando, P. Liu, L. F. Allard, and Y-P Sun, "Polymeric carbon nanocomposites from carbon nanotubes functionalized with matrix polymer," Macromolecules, vol. 36, no. 19, pp. 7199-7204, 2003.

[15] S. J. Park, M. S. Cho, S. T. Lim, H. J. Cho, and M. S. Jhon, "Synthesis and dispersion characteristics of multi-walled carbon nanotube composites with poly(methyl methacrylate) prepared by in situ bulk polymerization," Macromolecular Rapid Communications, vol. 24, no. 18, pp. 1070-1073, 2003.

[16] K. R. Yoon, W.-J. Kim, and I. S. Choi, "Functionalization of shortened single-walled carbon nanotubes with poly ( $p$ dioxanone) by "Grafting-From" approach," Macromolecular Chemistry and Physics, vol. 205, no. 9, pp. 1218-1221, 2004.

[17] D. Baskaran, J. W. Mays, and M. S. Bratcher, "Polymer-grafted multiwalled carbon nanotubes through surface-initiated polymerization," Angewandte Chemie International Edition, vol. 43, no. 16, pp. 2138-2142, 2004.

[18] H. Kong, C. Gao, and D. Yan, "Constructing amphiphilic polymer brushes on the convex surfaces of multi-walled carbon nanotubes by in situ atom transfer radical polymerization," Journal of Material Chemistry, vol. 14, no. 9, pp. 1401-1405, 2004.

[19] H. Kong, C. Gao, and D. Yan, "Controlled functionalization of multiwalled carbon nanotubes by in situ atom transfer radical polymerization," Journal of American Chemical Society, vol. 126, no. 2, pp. 412-413, 2004.

[20] G. Laruelle, J. Parvole, J. François, and L. Billon, "Block copolymer grafted-silica particles: a core/double shell hybrid inorganic/organic material," Polymer, vol. 45, no. 15, pp. 5013-5020, 2004.

[21] K. Karaky, E. Péré, C. Pouchan, J. Desbrières, C. Dérail, and L. Billon, "Effect of the synthetic methodology on molecular 
architecture: from statistical to gradient copolymers," Soft Matter, vol. 2, no. 9, pp. 770-778, 2006.

[22] G. Laruelle, J. François, and L. Billon, "Self-assembly in aqueous media of amphiphilic poly acrylic acid based di-block copolymers synthesized by direct nitroxide-mediated polymerization," Macromolecular Rapid Communications, vol. 25, no. 21, pp. 1839-1844, 2004.

[23] V. Datsyuk, C. Guerret-Piécourt, S. Dagréou, et al., "Double walled carbon nanotube/polymer composites via in situ nitroxide mediated polymerisation of amphiphilic block copolymers," Carbon, vol. 43, no. 4, pp. 873-876, 2005.

[24] L. Billon, V. Datsyuk, C. Guerret-Piécourt, and O. Guerret, "Composites materials base on carbon nanotubes and polymers matrices and process for obtaining same," WO Patent no. 108485, 2005.

[25] Y. Kang and T. A. Taton, "Micelle-encapsulated carbon nanotubes: a route to nanotube composites," Journal of American Chemical Society, vol. 125, no. 19, pp. 5650-5651, 2003.

[26] H. Kong, P. Luo, C. Gao, and D. Yan, "Polyelectrolytefunctionalized multiwalled carbon nanotubes: preparation, characterization and layer-by-layer self-assembly," Polymer, vol. 46, no. 8, pp. 2472-2485, 2005.

[27] E. Flahaut, R. R. Bacsa, A. Peigney, and C. Laurent, "Gramscale CCVD synthesis of doublewalled carbon nanotubes," Chemical Communication, no. 12, pp. 1442-1443, 2003.

[28] L. Couvreur, C. Lefay, J. Bellenay, B. Charleux, O. Guerret, and S. Magnet, "First nitroxide-mediated controlled free-radical polymerizaton of acrylic acid," Macromolecules, vol. 36, no. 22, pp. 8260-8267, 2003.

[29] M. S. P. Shaffer, X. Fan, and A. H. Windle, "Dispersion and packing of carbon nanotubes," Carbon, vol. 36, no. 11, pp. 1603-1612, 1998.

[30] L. Sonnenberg, J. Parvole, L. Billon, O. Borisov, M. Seitz, and H. E. Gaub, "AFM-based single molecule force spectroscopy of end-grafted poly(acrylic acid) monolayers," Macromolecules, vol. 39, no. 1, pp. 281-288, 2006.

[31] S. Tanodekaew, M. Prasitsilp, S. Swasdison, B. Thavornyutikarn, T. Pothsree, and R. Pateepasen, "Preparation of acrylic grafted chitin for wound dressing application," Biomaterials, vol. 25, no. 7-8, pp. 1453-1460, 2004.

[32] C. Lefay, J. Bellenay, B. Charleux, O. Guerret, and S. Magnet, "End-group characterization of poly(acrylic acid) prepared by nitroxide-mediated controlled free-radical polymerization," Macromolecular Rapid Communications, vol. 25, no. 13, pp. 1215-1220, 2004.

[33] D. Baskaran, J. W. Mays, and M. S. Bratcher, "Polymer adsorption in the grafting reactions of hydroxyl terminal polymers with multi-walled carbon nanotubes," Polymer, vol. 46, no. 14, pp. 5050-5057, 2005.

[34] O. Meincke, D. Kaempfer, H. Weickmann, C. Friedrich, M. Vathauer, and H. Warth, "Mechanical properties and electrical conductivity of carbon-nanotube filled polyamide- 6 and its blends with acrylonitrile/butadiene/styrene," Polymer, vol. 45, no. 3, pp. 739-748, 2004.

[35] T. Ogasawara, Y. Ishida, T. Ishikawa, and R. Yokota, "Characterization of multi-walled carbon nanotube/phenylethynyl terminated polyimide composites," Composites Part A: Applied Science and Manufacturing, vol. 35, no. 1, pp. 67-74, 2004.

[36] G. Stauffer, Introduction to Percolation Theory, Taylor \& Francis, London, UK, 1985. 

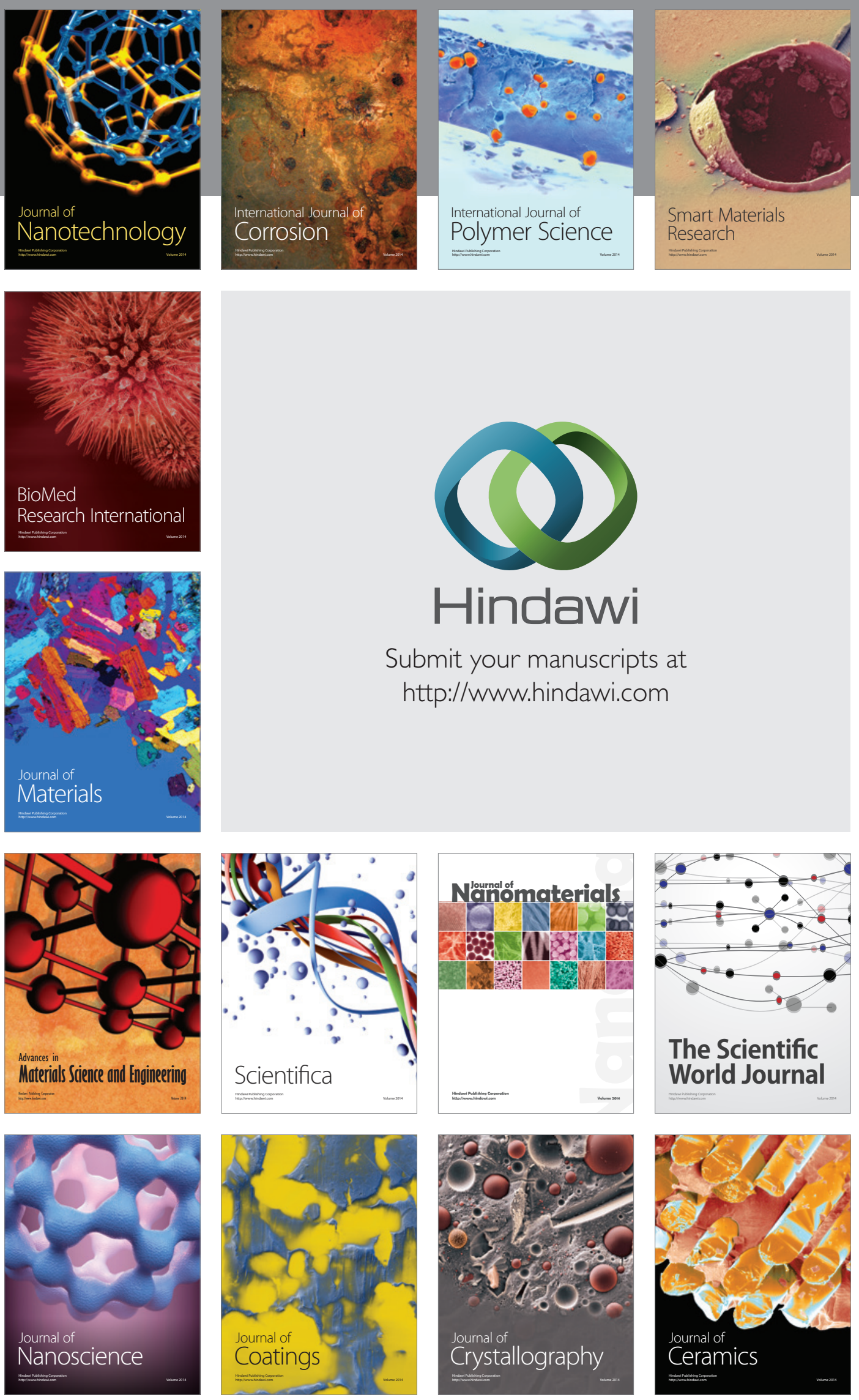

The Scientific World Journal

Submit your manuscripts at

http://www.hindawi.com

\section{World Journal}

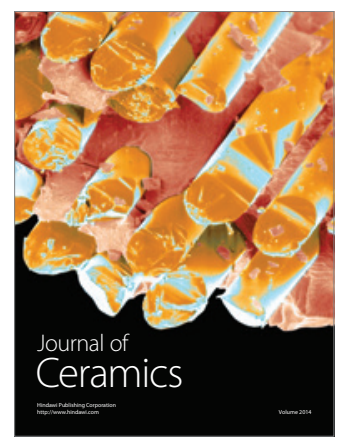

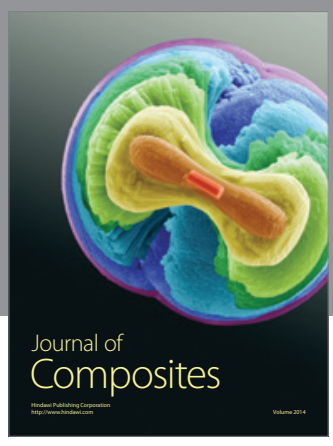
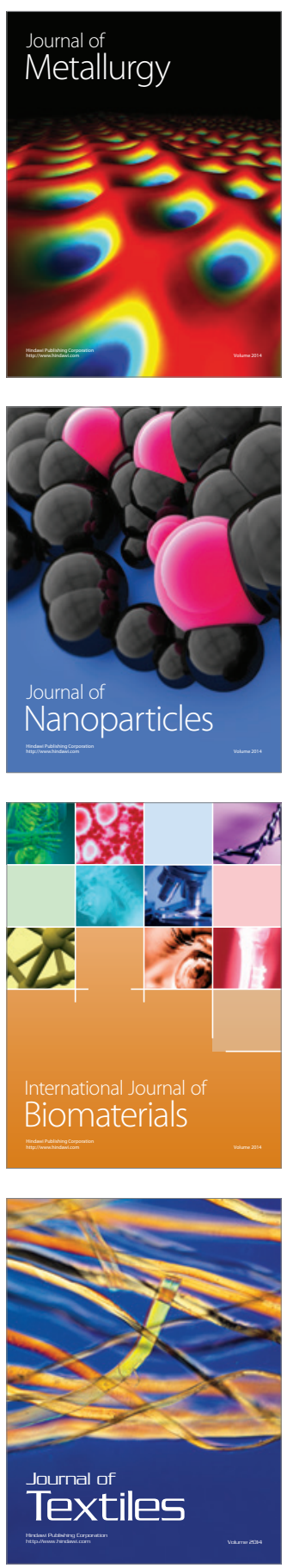